\title{
Safety and efficacy of sufentanil combined with midazolam in bronchoscopy under conscious sedation: retrospective study of 11,158 cases
}

\author{
Yao Yao ${ }^{1 \#}$, Zhuquan $\mathrm{Su}^{1 \#}$, Yu Chen ${ }^{1 \#}$, Yongshun Ye ${ }^{1 \#}$, Liya Lu ${ }^{2}$, Changhao Zhong ${ }^{1}$, Xiaobo Chen ${ }^{1}$, \\ Chunli Tang', Shiyue $\mathbf{L i}^{1}$
}

${ }^{1}$ State Key Laboratory of Respiratory Disease, National Clinical Research Center for Respiratory Disease, Guangzhou Institute of Respiratory Health, the First Affiliated Hospital of Guangzhou Medical University, Guangzhou 510120, China; ${ }^{2}$ Anesthesiology Department, the First Affiliated Hospital of Guangzhou Medical University, Guangzhou 510120, China

Contributions: (I) Conception and design: Y Yao, Y Chen, L Lu; (II) Administrative support: S Li; (III) Provision of study materials or patients: Y Ye, C Zhong, X Chen, C Tang; (IV) Collection and assembly of data: Y Yao; (V) Data analysis and interpretation: Y Yao, Z Su, Y Chen; (VI) Manuscript writing: All authors; (VII) Final approval of manuscript: All authors.

"These authors contributed equally as first authors.

Correspondence to: Shiyue Li. State Key Laboratory of Respiratory Disease, National Clinical Research Center for Respiratory Disease, Guangzhou Institute of Respiratory Health, the First Affiliated Hospital of Guangzhou Medical University, No. 151Yanjiang Road, Guangzhou 510120, China. Email: lishiyue@188.com.

Background: The recommended conscious sedation for bronchoscopy is still lacking. The safety and efficacy of sufentanil combined with midazolam in bronchoscopy under conscious sedation is not well elucidated.

Methods: A retrospective analysis was conducted on patients who received bronchoscopy in the First Affiliated Hospital of Guangzhou Medical University from September 2013 to July 2017. Sufentanil and midazolam were administrated for conscious sedation. The drug dosage, sedating effect and adverse event were collected and analyzed.

Results: Totally, 7,089 males and 4,069 females aged 54 \pm 16 years (ranged from 4 to 94 years) were enrolled in this study. The dosage of sufentanil and midazolam were $5.25 \pm 1.28 \mathrm{mcg}(2-13 \mathrm{mcg})$ and $2.03 \pm 0.51 \mathrm{mg}$ $(0.5-4.5 \mathrm{mg})$, respectively. Ninety-eight point six percent $(10,998 / 11,158)$ of bronchoscopies were successfully completed, while $68.7 \%(7,670 / 11,158)$ procedures were performed with initial dose of 5 mcg sufentanil and $2 \mathrm{mg}$ midazolam. Endobronchial biopsy, transbronchial lung biopsy (TBLB), transbronchial needle aspiration (TBNA), therapeutic procedure and asthma were predictors of giving incremental doses of sufentanil and midazolam (all OR $>1, \mathrm{P}<0.05$ ), whereas, the age was associated with lower incidence of adding dose of sufentanil and midazolam (both $\mathrm{OR}<1, \mathrm{P}<0.05$ ). Patients with chronic obstructive lung disease (COPD) had lower incidence of adding dose of midazolam alone ( $\mathrm{OR}=0.597, \mathrm{P}=0.003)$. Whereas, female and pulmonary infection were predictors of adding dose of sufentanil alone $(\mathrm{OR}>1, \mathrm{P}<0.05)$. The conscious sedation related adverse events were not observed.

Conclusions: Sufentanil combined with midazolam was safe and effective for bronchoscopy under conscious sedation.

Keywords: Conscious sedation; midazolam; sufentanil; bronchoscopy

Submitted May 09, 2019. Accepted for publication Sep 30, 2019.

doi: $10.21037 /$ jtd.2019.10.03

View this article at: http://dx.doi.org/10.21037/jtd.2019.10.03

(c) Journal of Thoracic Disease. All rights reserved. 


\section{Introduction}

Analgesia and sedation are guarantees for successfully performing diagnostic flexible bronchoscopy. Sedative has the role of alleviating the anxiety and stress, contributing to conscious sedation and improving the comfort and cooperation. Analgesics could help relieve the pain during the procedure of bronchoscopy (1). The international guidelines recommend to that midazolam should be used in combination with opioid (fentanyl, alfentanil, etc.) to improve the patient's tolerance (2,3). Although sufentanil is 10 times more potent and has fewer side effect than fentanyl (4), the application of sufentanil in bronchoscopy have been reported in a few studies (5-7). The safety and efficacy of the combination of sufentanil and midazolam for conscious sedation are not well elucidated. Hence, we have conducted a large-scale systematic retrospective study of analyzing 11,158 cases of bronchoscopy with sufentanil and midazolam for conscious sedation.

\section{Methods}

\section{Subjects}

Patients who received bronchoscopy, with administrating sufentanil and midazolam for conscious sedation in the First Affiliated Hospital of Guangzhou Medical University from September 2013 to July 2017 were included in this study. The drug dosage, sedating effect and adverse event were collected and analyzed. The paper reports and electronic medical records were reviewed. This study was approved by the Ethics Committee of the First Affiliated Hospital of Guangzhou Medical University. The study has been retrospectively registered in ClinicalTrials.gov on March 24, 2019 (Registry ID: NCT03890094).

\section{Protocol}

Before the bronchoscopy, oxygen supplementation was given through the nasal cannula (2-5 L/min, adjusted as needed). $2 \%$ lidocaine was applied for topical anesthesia, whilst heart rate, electrocardiogram and pulse oxygen saturation $\left(\mathrm{SpO}_{2}\right)$ were monitored. Afterward, midazolam (2 mg) and sufentanil $(5 \mathrm{mcg})$ were intravenous administrated for conscious sedation, the initial dose of midazolam would be decreased by $0.5-1.0 \mathrm{mg}$, and sufentanil be decreased by 1-2 mcg if circumstances were as following: weight $<50 \mathrm{~kg}$, liver or kidney dysfunction, cardiac or pulmonary dysfunction, malnutrition or cachexia. Furthermore, the initial dose of midazolam would be increased by $0.5-1 \mathrm{mg}$, and sufentanil be increased by $1-2 \mathrm{mcg}$ as weight $>70 \mathrm{~kg}$, history of psychotropic drugs uses and alcoholism or poor tolerance in previous bronchoscopy. Age, gender, underlying diseases, the total dosage of sufentanil and midazolam, diagnostic and therapeutic procedures, adverse events were recorded and analyzed.

\section{Statistical analysis}

All data were analyzed using statistical software SPSS version 22.0 (SPSS Inc., Chicago, IL, USA). The distribution of variables was assessed by means of a Kolmogorov-Smirnov goodness-of-fit test. Continuous variables were expressed as mean $\pm \mathrm{SD}$ or medians and interquartile ranges (IQRs) according to distribution, whereas categorical variables were expressed as proportions. Groups were compared with unpaired $t$-test, MannWhitney test, chi-square test or Fisher exact test as appropriate. Multivariable logistic regression analyses were applied to identify risk factors of failure in bronchoscopy, severe cough and adding dosage. Variable screening method was forward stepwise regression method based on maximum likelihood estimation (Forward: LR method). The Wald $\chi^{2}$ test was used to estimate the regression parameters, and the likelihood ratio test was used to estimate the model fitting. $\mathrm{P}<0.05$ was considered statistically significant.

\section{Results}

Totally, 11,158 cases including 7,089 males and 4,069 females aged $54 \pm 16$ years (ranged from 4 to 94 years) were analyzed in this study; $34.66 \%$ cases $(3,867 / 11,158)$ were outpatients, while $7.37 \%(285 / 3,867)$ were being hospitalization. No outpatients were hospitalized due to adverse events after bronchoscopy; $89.71 \%(10,010 / 11,158)$ cases received diagnostic bronchoscopy, and $10.75 \%(1,199 / 11,158)$ was treated with therapeutic procedures (Table 1).

\section{Dosage}

The mean dosage of sufentanil and midazolam were $5.25 \pm 1.28 \mathrm{mcg}(2-13 \mathrm{mcg})$ and $2.03 \pm 0.51 \mathrm{mg}(0.5-4.5 \mathrm{mg})$, respectively; $68.74 \%(7,670 / 11,158)$ patients were only administered initial dose of $5 \mathrm{mcg}$ sufentanil and $2 \mathrm{mg}$ midazolam during the procedure. A total of 221 patients $(221 / 11,158,1.98 \%)$ and 3 patients $(3 / 11,158,0.03 \%)$ needed additional sufentanil or midazolam during 
Table 1 The diagnostic and therapeutic procedures of study subjects

\begin{tabular}{|c|c|c|c|c|c|}
\hline Diagnostic procedure & Case & Proportion & Therapeutic procedure & Case & Proportion \\
\hline Biopsy & 4,585 & $41.1 \%$ & Cryotherapy & 212 & $1.9 \%$ \\
\hline Brushing & 3,970 & $35.6 \%$ & Laser & 72 & $0.6 \%$ \\
\hline EBUS-GS & 1,191 & $10.7 \%$ & Electrocoagulation/electrosection & 63 & $0.6 \%$ \\
\hline BAL & 1,067 & $9.6 \%$ & Metal stent placement & 55 & $0.5 \%$ \\
\hline \multirow[t]{3}{*}{ c-TBNA } & 175 & $1.6 \%$ & Foreign body removal & 53 & $0.5 \%$ \\
\hline & & & Snare & 39 & $0.3 \%$ \\
\hline & & & APC & 36 & $0.3 \%$ \\
\hline
\end{tabular}

EBUS-GS, endobronchial ultrasound with guide sheath; EBUS-TBNA, endobronchial ultrasound-guided transbronchial needle aspiration; BAL, bronchoalveolar lavage; c-TBNA, conventional transbronchial needle aspiration; APC, argon plasma coagulation.

bronchoscopy procedure. Furthermore, $11.61 \%$ patients $(1,296 / 11,158)$ needed extra sufentanil and midazolam during the procedure.

Adding dose of sufentanil was associated with increased rates of female ( $41.3 \%$ vs. $35.7 \%, \mathrm{P}<0.001)$, younger patients (54 vs. 57 years, $\mathrm{P}<0.001$ ), increased rates of therapeutic procedure $(19.0 \%$ vs. $9.5 \%, \mathrm{P}<0.001)$, biopsy $(48.8 \%$ vs. $39.9 \%, \mathrm{P}<0.001)$, transbronchial needle aspiration (TBNA) (18.7\% vs. $9.0 \%, \mathrm{P}<0.001)$, increased rates of patients with asthma $(3.1 \%$ vs. $1.8 \%, \mathrm{P}=0.001)$ and decrease of chronic obstructive lung disease (COPD) (3.4\% vs. 5.5\%, $\mathrm{P}<0.001$ ) (Table 2). Moreover, adding dose of midazolam was associated with increased rates of female $(39.9 \%$ vs. $36.0 \%, \mathrm{P}=0.007$ ), younger patients ( 54 vs. 57 years, $\mathrm{P}<0.001)$, increased rates of therapeutic procedure $(17.9 \%$ vs. $9.8 \%, \mathrm{P}<0.001)$, biopsy $(48.4 \%$ vs. $40.2 \%, \mathrm{P}<0.001)$, TBNA $(19.5 \%$ vs. $9.1 \%, \mathrm{P}<0.001)$ and decreased rates of patients with COPD (2.9\% vs. $5.5 \%, \mathrm{P}<0.001)$.

\section{Safety}

Oxygen saturation repeatedly decreased lower than $80 \%$ during procedure in 15 cases, which led to the termination of bronchoscopy. However, none of them had respiratory failure requiring tracheal intubation, or cardiovascular and nervous adverse events. Generally, hemoptysis present in 571 cases $(5.1 \%)$, while pneumothorax requiring tube thoracostomy occurred in 63 cases $(0.6 \%)$ after the procedure. They all had been fully recovered in one week (Table 3).

\section{Efficacy}

Bronchoscopies were successfully performed in $98.6 \%$ cases $(10,998 / 11,158)$. The procedure was taking $10-30 \mathrm{~min}$ per case. However, 162 patients (1.4\%) failed to complete the procedure, which attributed to severe cough $(112 / 11,158)$, hypoxemia $(15 / 11,158)$, poor cooperation during bronchoscopy $(2 / 11,158)$. Failure of bronchoscopy was associated with increased rates of TBNA procedure (17.9\% vs. $11.1 \%, \mathrm{P}=0.001)$, patients with asthma $(6.2 \%$ vs. $2.1 \%, \mathrm{P}<0.001)$ and interstitial lung disease (ILD) $(4.9 \%$ vs. $1.3 \%, \mathrm{P}<0.001)$ (Table 4). Severe cough was associated with decreased rates of therapeutic procedure $(2.7 \% \mathrm{vs}$. $10.8 \%, \mathrm{P}=0.006)$, increased rates of asthma ( $8.0 \%$ vs. $1.9 \%$, $\mathrm{P}<0.001)$, ILD (6.3\% vs. $1.2 \%, \mathrm{P}<0.001)$ and pulmonary infection $(17.0 \%$ vs. $7.8 \%, \mathrm{P}<0.001)$.

\section{Predictors of failure in bronchoscopy, severe cough and adding dose of sufentanil and midazolam}

Logistic regression analysis was applied to identify the predictors of failure in bronchoscopy, severe cough and additional dose of sufentanil and midazolam. Variables included: age, gender, initial dose of midazolam and sufentanil, type of procedure (biopsy, BAL, TBNA, 
Table 2 Patients' characteristics stratified by adding dose of sufentanil and midazolam

\begin{tabular}{|c|c|c|c|c|c|c|}
\hline \multirow[t]{2}{*}{ Characteristics } & \multicolumn{3}{|c|}{$\begin{array}{l}\text { Adding dose of sufentanil, } \\
\text { median [IQRs] or number (\%) }\end{array}$} & \multicolumn{3}{|c|}{$\begin{array}{l}\text { Adding dose of midazolam, } \\
\text { median [IQRs] or number (\%) }\end{array}$} \\
\hline & Yes $(n=1,517)$ & No $(n=9,641)$ & $P$ value & Yes $(n=1,299)$ & No $(n=9,859)$ & $P$ value \\
\hline Female & $627(41.3)$ & $3,442(35.7)$ & $<0.001$ & 518 (39.9) & $3,551(36.0)$ & 0.007 \\
\hline Age & $54[42-64]$ & 57 [43-65] & $<0.001$ & 54 [43-63] & 57 [43-65] & $<0.001$ \\
\hline Therapeutic procedure & $288(19.0)$ & $912(9.5)$ & $<0.001$ & 233 (17.9) & $967(9.8)$ & $<0.001$ \\
\hline Biopsy & $740(48.8)$ & 3,848 (39.9) & $<0.001$ & $629(48.4)$ & 3,959 (40.2) & $<0.001$ \\
\hline BAL & $143(9.4)$ & $925(9.6)$ & 0.836 & $112(8.6)$ & $956(9.7)$ & 0.216 \\
\hline Lung malignancies & $88(5.8)$ & $494(5.1)$ & 0.270 & $82(6.3)$ & $500(5.1)$ & 0.059 \\
\hline Airway stenosis & $66(4.4)$ & $470(4.9)$ & 0.375 & $55(4.2)$ & $481(4.9)$ & 0.307 \\
\hline ILD & $13(0.9)$ & $124(1.3)$ & 0.158 & $10(0.8)$ & $127(1.3)$ & 0.111 \\
\hline Pulmonary infection & $126(8.3)$ & $750(7.8)$ & 0.478 & $104(8.0)$ & $772(7.8)$ & 0.825 \\
\hline
\end{tabular}

IQRs, interquartile ranges; BAL, bronchoalveolar lavage; TBNA, transbronchial needle aspiration; COPD, chronic obstructive lung disease; ILD, interstitial lung disease.

Table 3 Adverse events during and after bronchoscopy

\begin{tabular}{lrc}
\hline Adverse events & Cases & Percentage \\
\hline Hypoxemia & 15 & $0.1 \%$ \\
Severe cough & 112 & $1.0 \%$ \\
Poor cooperation & 2 & $0.0 \%$ \\
Hemoptysis & 571 & $5.1 \%$ \\
Pneumothorax & 63 & $0.6 \%$ \\
Total & 763 & $6.8 \%$ \\
\hline
\end{tabular}

Hypoxemia: $\mathrm{SpO}_{2}$ decreased to lower than $80 \%$ during procedure. Pneumothorax: pneumothorax requiring tube thoracostomy.

therapeutic procedure), as well as underlying disease (asthma, COPD, ILD, lung malignancies, pulmonary infection, airway stenosis).

The independent predictors of bronchoscopy failure were ILD, asthma, pulmonary infection, TBNA, and higher initial dose of sufentanil $(\mathrm{OR}>1, \mathrm{P}<0.05)$, but not age, gender, initial dose of midazolam, performance of biopsy, $\mathrm{BAL}$ and therapeutic procedure, COPD, lung malignancies and airway stenosis $(\mathrm{P}>0.05)$ (Table 5).

ILD, asthma, pulmonary infection and higher initial dose of sufentanil $(\mathrm{OR}>1, \mathrm{P}<0.05)$ were risk factors of severe cough (Table 5). Whereas, performance of therapeutic procedure contributed to a protective factor $(\mathrm{OR}=0.221$, $\mathrm{P}=0.011$ ).

The logistic regression analysis demonstrated that the predictors of adding dose of sufentanil and midazolam were performance of biopsy, TBNA and therapeutic procedure and asthma $(\mathrm{OR}>1, \mathrm{P}<0.05)$. However, the age was a protective factor of additional dose of sufentanil and midazolam $(\mathrm{OR}<1, \mathrm{P}<0.05)$, while $\mathrm{COPD}$ was a protective factor adding dose of midazolam $(\mathrm{OR}=0.597$, $\mathrm{P}=0.003)$. Furthermore, female, pulmonary infection and higher initial dose of midazolam were risk factors of adding dose of sufentanil $(\mathrm{OR}>1, \mathrm{P}<0.05)$. Higher initial dose of sufentanil was protective factor of adding dose of sufentanil $(\mathrm{OR}=0.737, \mathrm{P}<0.05)($ Table 6$)$. 
Table 4 Patients' characteristics stratified by completion of bronchoscopy and severe cough

\begin{tabular}{|c|c|c|c|c|c|c|}
\hline \multirow[t]{2}{*}{ Characteristics } & \multicolumn{3}{|c|}{$\begin{array}{l}\text { Completion of bronchoscopy, } \\
\text { median [IQRs] or number (\%) }\end{array}$} & \multicolumn{3}{|c|}{$\begin{array}{c}\text { Severe cough, } \\
\text { median [IQRs] or number (\%) }\end{array}$} \\
\hline & Yes $(n=10,149)$ & No $(n=162)$ & $P$ value & Yes $(n=112)$ & No $(n=11,046)$ & $P$ value \\
\hline Age & 57 [43-65] & 58 [48-64] & 0.790 & 59 [48-66] & 57 [43-65] & 0.229 \\
\hline Therapeutic procedure & $1,188(11.7)$ & $12(7.4)$ & 0.166 & $3(2.7)$ & $1,197(10.8)$ & 0.006 \\
\hline Biopsy & $4,522(44.6)$ & $66(40.7)$ & 0.922 & $45(40.2)$ & $4,543(41.1)$ & 0.839 \\
\hline BAL & $1,050(10.3)$ & $18(11.1)$ & 0.502 & $16(14.3)$ & $1,052(9.5)$ & 0.088 \\
\hline Lung malignancies & $573(5.6)$ & $9(5.6)$ & 0.845 & $9(8.0)$ & $573(5.2)$ & 0.177 \\
\hline Airway stenosis & $531(5.2)$ & $5(3.1)$ & 0.303 & $5(4.5)$ & $531(4.8)$ & 0.866 \\
\hline ILD & $129(1.3)$ & $8(4.9)$ & $<0.001$ & $7(6.3)$ & $130(1.2)$ & $<0.001$ \\
\hline Pulmonary infection & $853(8.4)$ & $23(14.2)$ & 0.002 & $19(17.0)$ & $857(7.8)$ & $<0.001$ \\
\hline
\end{tabular}

IQRs, interquartile ranges; BAL, bronchoalveolar lavage; TBNA, transbronchial needle aspiration; COPD, chronic obstructive lung disease; ILD, interstitial lung disease.

Table 5 Predictors of failure in bronchoscopy and severe cough

\begin{tabular}{|c|c|c|c|c|c|c|}
\hline Variable & \multicolumn{3}{|c|}{ Failure in bronchoscopy } & \multicolumn{3}{|c|}{ Severe cough } \\
\hline Initial dose of sufentanil & 1.393 & $1.161-1.672$ & $<0.001$ & 1.681 & $1.390-2.033$ & $<0.001$ \\
\hline TBNA & 2.156 & $1.427-3.258$ & $<0.001$ & - & - & 0.346 \\
\hline Pulmonary infection & 2.144 & $1.364-3.371$ & 0.001 & 2.422 & $1.462-4.011$ & 0.001 \\
\hline ILD & 5.187 & $2.477-10.865$ & $<0.001$ & 5.996 & $2.713-13.251$ & $<0.001$ \\
\hline Therapeutic procedure & - & - & 0.369 & 0.221 & $0.069-0.703$ & 0.011 \\
\hline
\end{tabular}

TBNA, transbronchial needle aspiration; ILD, interstitial lung disease.

\section{Discussion}

In this study, we had retrospectively analyzed the administration of sufentanil and midazolam for conscious sedation during bronchoscopy. The results indicated the combination medication was safe and effective for bronchoscopy. $98.6 \%$ of the patients were successfully received bronchoscopy, no severe complication occurred during the procedure.

Midazolam has been the first choice and basic medication for bronchoscopy for fast acting, reversibility and the retrograde amnesia effect. Opioids could bind with opioid receptors for activating its signaling pathways (8). The activation of opioid downstream reduces calcium influx and activates presynaptic opioid receptors, leading to the 
Table 6 Predictors of adding dose of sufentanil and midazolam

\begin{tabular}{|c|c|c|c|c|c|c|}
\hline Variable & \multicolumn{3}{|c|}{ Sufentanil } & \multicolumn{3}{|c|}{ Midazolam } \\
\hline Age & 0.994 & $0.991-0.998$ & 0.001 & 0.993 & $0.989-0.997$ & $<0.001$ \\
\hline Female & 1.227 & $1.093-1.377$ & 0.001 & - & - & 0.087 \\
\hline Initial dose of sufentanil & 0.737 & $0.630-0.862$ & $<0.001$ & - & - & 0.242 \\
\hline Biopsy & 2.106 & $1.860-2.385$ & $<0.001$ & 1.977 & $1.734-2.253$ & $<0.001$ \\
\hline TBNA & 3.374 & $2.880-3.953$ & $<0.001$ & 3.311 & 2.812-3.899 & $<0.001$ \\
\hline Therapeutic procedure & 3.634 & $3.072-4.300$ & $<0.001$ & 3.201 & $2.679-3.824$ & $<0.001$ \\
\hline Pulmonary infection & 1.301 & $1.063-1.594$ & 0.011 & - & - & 0.094 \\
\hline
\end{tabular}

TBNA, transbronchial needle aspiration; COPD, chronic obstructive lung disease.

reduction of carrier material release. Moreover, opioid increasing the post-synaptic membrane potassium ion flux might reduce the neuron excitability. Inhibition of nociceptive stimulation transmission contributes to the basis of opioid analgesic effect (9). Although Fentanyl has been widely used for fast acting, it might cause asthma acute exacerbation (10).

Sufentanil, which was synthesized in 1974, has higher lipophilicity, potency and affinity to opioid receptor compared with those of fentanyl. Furthermore, sufentanil has lower incidence of nausea and vomiting, weaker respiratory depression, which could be antagonized by naloxone (11), than those of fentanyl. Hence, the pharmacokinetics of sufentanil makes it suitable for conscious sedation during bronchoscopy.

In the current study, the initial dose of midazolam was set to $2 \mathrm{mg}$, according to the report that the average weight of Chinese adult males was $66.2 \mathrm{~kg}$, the average weight of adult females was $57.3 \mathrm{~kg}$. The dosage for males was 0.030 and $0.035 \mathrm{mg} / \mathrm{kg}$ for females, which was determined based on the recommendation of British guidelines that no more than $5 \mathrm{mg}$ for patients under the age of 70 years ( $2 \mathrm{mg}$ midazolam for patients over 70 years) initially (3). As the synergistic effect of benzodiazepines and opioids on patient's tolerance, the initial dose of sufentanil was $5 \mathrm{mcg}(0.076 \mathrm{mcg} / \mathrm{kg}$ for males and $0.088 \mathrm{mcg} / \mathrm{kg}$ for females), which was lower than those in the previous studies $(0.1-0.2 \mathrm{mcg} / \mathrm{kg})(12,13) .70 \%$ of patients were applied an initial dose of $5 \mathrm{mcg}$ sufentanil and $2 \mathrm{mg}$ midazolam during the procedure, indicating that the dosage was suitable for most of the Chinese patients.

The regression analysis demonstrated that the age, gender, underlying diseases, type of procedure, and initial dose might be the predictors of midazolam and sufentanil dosage. The older patients had the lower the incidence of adding dose of midazolam and sufentanil during bronchoscopy. Female gender was one of the risk factors of higher dosage of sufentanil, which might be explained that females have higher hyperalgesia, more sensitive to noxious simulation, and weaker response to analgesics (14). Lower dosage of midazolam was administrated in patients with COPD for the lung function decline and the risk of respiratory depression. Furthermore, the higher initial dose of midazolam might induce extra dose of sufentanil, which might depend upon the consideration of the patients' history of psychotropic substance usage, habitual alcohol consumption and the poor tolerance of the previous bronchoscopy.

Patients with asthma and interstitial lung disease had higher incidence of severe cough during bronchoscopy, but COPD, lung malignancies and airway stenosis had no significant effect on the occurrence of severe cough. The initial sufentanil dose might contribute to a risk factor of severe cough and failure in bronchoscopy. It was reported that cough is one of the most common complications of opioids, which is called opioid-induced cough (OIC) (12). Agarwal et al. found that $15 \%(26 / 165)$ of the patients who received sufentanil $0.3 \mathrm{mcg} / \mathrm{kg}$ intravenously over $5 \mathrm{~s}$ 
had cough symptom, whereas, the severity and incidence of coughing with sufentanil were less than those with equipotent bolus of fentanyl (15). Although the application of opioids might induce cough symptom, it would be weakened as combined with topical anesthesia or sedative.

Sufentanil might induce respiratory depression by attenuating the sensitivity of the respiratory center to $\mathrm{CO}_{2}$, reducing respiratory rate and tidal volume (16). Midazolam acts on the brainstem reticular structure and the limbic system through benzodiazepine receptors, increasing upper airway and lung resistance and resulting central respiratory depression (17). In this study, $0.16 \%$ patients had $\mathrm{O}_{2}$ desaturation due to respiratory depression, which led to the termination of bronchoscopy. These patients had underlying diseases, such as ventilatory dysfunction, respiratory failure and hypoproteinemia. In order to avoid respiratory depression, patients with cardiopulmonary disease should be applied with lower dosage of sufentanil and midazolam. As such, there was no respiratory failure requiring endotracheal intubation in our study.

Some limitations should be taken into consideration. First, it was a retrospective study, we did not analyze patients' tolerance, physicians' satisfaction or nervous system symptoms induced by sufentanil or midazolam. Second, we had no comparisons of sufentanil with other opioids, and the determination of the optimal dosage was absent. Furthermore, a prospective, randomized, doubleblind clinical trial of combination of sufentanil and midazolam is needed in the further study.

In conclusion, sufentanil combined with midazolam was safe and effective for bronchoscopy under conscious sedation. Age, gender, underlying diseases, diagnostic and therapeutic procedures might have contributed to the predictors of the requirement dosage of sufentanil and midazolam.

\section{Acknowledgments}

The authors thank Dr. Wu and Dr. Wang (State Key Laboratory of Respiratory Disease, National Clinical Research Center for Respiratory Disease, Guangzhou Institute of Respiratory Health, the First Affiliated Hospital of Guangzhou Medical University) for their recommendations and English language editing.

Funding: This work was supported by the National Natural Science Foundation of China (No. 81770017) and the Youth Fund of Sate Key Laboratory of Respiratory Disease (SKLRD-QN-201914).

\section{Footnote}

Conflicts of Interest: The authors have no conflicts of interest to declare.

Ethical Statement: The authors are accountable for all aspects of the work in ensuring that questions related to the accuracy or integrity of any part of the work are appropriately investigated and resolved. This retrospective study was approved by the ethics committee of The First affiliated Hospital of Guangzhou Medical University (201815), and it has been retrospectively registered ClinicalTrials.gov on March 24, 2019 (Registry ID: NCT03890094). The study outcomes will not affect the future management of the patients. Patient's personal data have been secured.

\section{References}

1. Matot I, Kramer MR. Sedation in outpatient bronchoscopy. Respir Med 2000;94:1145-53.

2. Du Rand IA, Blaikley J, Booton R, et al. British Thoracic Society guideline for diagnostic flexible bronchoscopy in adults: accredited by NICE. Thorax 2013;68 Suppl 1:11-44.

3. Wahidi MM, Jain P, Jantz M, et al. American College of Chest Physicians consensus statement on the use of topical anesthesia, analgesia, and sedation during flexible bronchoscopy in adult patients. Chest 2011;140:1342-50.

4. Maciejewski D. Sufentanil in anaesthesiology and intensive therapy. Anaesthesiol Intensive Ther 2012;44:35-41.

5. Yuan Y, Zhang J, Yue HL, et al. A preliminary study of different methods of anesthesia for painless bronchoscopy. Zhonghua Jie He He Hu Xi Za Zhi 2019;42:106-13.

6. Dang X, Hu W, Yang Z, et al. Dexmedetomidine plus sufentanil for pediatric flexible bronchoscopy: A retrospective clinical trial. Oncotarget 2017;8:41256-64.

7. Ji M, Tao J, Cheng M, et al. Endotracheal Administration of Sufentanil and Tetracaine During Awake Fiberoptic Intubation. Am J Ther 2016;23:e92-7.

8. Christie MJ, Connor M, Vaughan CW, et al. Cellular actions of opioids and other analgesics: implications for synergism in pain relief. Clin Exp Pharmacol Physiol 2000;27:520-3.

9. Jordan B, Devi LA. Molecular mechanisms of opioid receptor signal transduction. Br J Anaesth 1998;81:12-9.

10. Parmar MS. Exacerbation of asthma secondary to fentanyl transdermal patch. BMJ Case Rep 2009. doi: 10.1136/ bcr.10.2008.1062. 
11. Howie MB, Smith DF, Reilley TE, et al. Postoperative course after sufentanil or fentanyl anesthesia for coronary artery surgery. J Cardiothorac Vasc Anesth 1991;5:485-9.

12. Sun L, Guo R, Sun L. The impact of prophylactic intravenous lidocaine on opioid-induced cough: a meta-analysis of randomized controlled trials. J Anesth 2014;28:325-33.

13. Agarwal A, Azim A, Ambesh S, et al. Salbutamol, beclomethasone or sodium chromoglycate suppress coughing induced by iv fentanyl. Can J Anaesth 2003;50:297-300.

14. Palmeira CC, Ashmawi HA, Posso Ide P. Sex and pain perception and analgesia. Rev Bras Anestesiol

Cite this article as: Yao Y, Su Z, Chen Y, Ye Y, Lu L, Zhong C, Chen X, Tang C, Li S. Safety and efficacy of sufentanil combined with midazolam in bronchoscopy under conscious sedation: retrospective study of 11,158 cases. J Thorac Dis 2019;11(10):4127-4134. doi: 10.21037/jtd.2019.10.03
2011;61:814-28.

15. Agarwal A, Gautam S, Nath SS, et al. Comparison of the incidence and severity of cough induced by sufentanil and fentanyl: a prospective, randomised, double-blind study. Anaesthesia 2007;62:1230-2.

16. Boom M, Niesters M, Sarton E, et al. Non-analgesic effects of opioids: opioid-induced respiratory depression. Curr Pharm Des 2012;18:5994-6004.

17. Oshima H, Nakamura M, Watanabe O, et al. Dexmedetomidine provides less body motion and respiratory depression during sedation in doubleballoon enteroscopy than midazolam. SAGE Open Med 2017;5:2050312117729920. 